\title{
Bilateral Generalization of Fifth and Eighth Order Mock Theta Functions
}

\author{
Sameena Saba \\ Mathematics Department, Lucknow University, India
}

Abstract: We generalize fifth order mock theta functions of Ramanujan and eighth order mock theta functions of Gordon and McIntosh. We show they are $F_{q}$-functions and give their alternative definition. We give expansion formula and give relationship among these functions.

2000 Mathematics Subject Classification.33D15

Keywords: Bilateral series, mock theta functions.

\section{Introduction}

The fifth order mock theta functions of Ramanujan were considered by Watson [1] and Andrews [2]. Gordon and McIntosh [3] developed eight mock theta functions and called them of order eight, but later four of them were found of lower order.

The idea of this paper is to generalize these fifth and eighth order mock functions and consider their bilateral form. In bilateral form we take the summation of the defining series from $-\infty$ to $\infty$. Watson called them 'Complete'.

\section{We divide the paper as follows:}

In section 6, we show that these functions are $F_{q}$-functions. In section 7, using Bailey's transform we give an alternative definition and in section 8 , show relationship between generalized bilateral eighth order mock theta functions and generalized bilateral third order mock theta functions.

In section 9, we use Slater's expansion formula to express these generalized functions as a bilateral series and in section 10, show the relationship among themselves.

\section{Notations and symbol}

We shall use the following usual basic hypergeometric notations:

For $\left|q^{k}\right|<1$,

$$
\begin{aligned}
& \left(a ; q^{k}\right)_{n}=(1-a)\left(1-a q^{k}\right) \cdots\left(1-a q^{k(n-1)}\right), \quad n \geq 1 \\
& (a)_{n}=(a ; q)_{n} \text {, } \\
& (a)_{0}=1 \text {, } \\
& (a ; q)_{\infty}=\prod_{j=0}^{\infty}\left(1-a q^{j}\right), \\
& \left(a_{1}, a_{2}, \cdots, a_{m} ; q\right)_{n}=\left(a_{1} ; q\right)_{n}\left(a_{2} ; q\right)_{n} \cdots\left(a_{m} ; q\right)_{n} . \\
& { }_{r} \psi_{r}\left[\begin{array}{l}
a_{1}, a_{2}, \cdots, a_{r} \\
\left.b_{1}, b_{2}, \cdots, b_{r} ; q ; z\right]
\end{array}\right]=\sum_{n=-\infty}^{\infty} \frac{\left(a_{1}, a_{2}, \cdots, a_{r} ; q\right)_{n}}{\left(b_{1}, b_{2}, \cdots, b_{r} ; q\right)_{n}} z^{n} .
\end{aligned}
$$

where $\left|b_{1} b_{2} \cdots b_{r} / a_{1} a_{2} \cdots a_{r}\right|<|z|<1$.

III. Definition of mock theta functions of order third, five and eight

The third order mock theta functions of Ramanujan:

$$
\begin{aligned}
f(q)=\sum_{n=0}^{\infty} \frac{q^{n^{2}}}{(-q)_{n}^{2}}, & \phi(q)=\sum_{n=0}^{\infty} \frac{q^{n^{2}}}{\left(-q^{2} ; q^{2}\right)_{n}}, \\
\psi(q)=\sum_{n=1}^{\infty} \frac{q^{n^{2}}}{\left(q ; q^{2}\right)_{n}}, & \chi(q)=\sum_{n=0}^{\infty} \frac{q^{n^{2}}}{\left(1-q+q^{2}\right) \ldots \ldots\left(1-q^{n}+q^{2 n}\right)}
\end{aligned}
$$


$\omega(q)=\sum_{n=0}^{\infty} \frac{q^{2 n(n+1)}}{\left(q ; q^{2}\right)_{n+1}^{2}}$

$v(q)=\sum_{n=0}^{\infty} \frac{q^{n(n+1)}}{\left(-q ; q^{2}\right)_{n+1}}$

and

$$
\rho(q)=\sum_{n=0}^{\infty} \frac{q^{2 n(n+1)}}{\left(1+q+q^{2}\right) \ldots \ldots\left(1+q^{2 n+1}+q^{4 n+2}\right)} .
$$

The fifth order mock theta functions of Ramanujan [4]:

$$
\begin{array}{rlrl}
f_{0}(q)=\sum_{n=0}^{\infty} \frac{q^{n^{2}}}{(-q ; q)_{n}}, & \phi_{0}(q) & =\sum_{n=0}^{\infty} q^{n^{2}}\left(-q ; q^{2}\right)_{n}, \\
\psi_{0}(q)=\sum_{n=0}^{\infty} q^{\frac{(n+1)(n+2)}{2}}(-q ; q)_{n}, & F_{0}(q) & =\sum_{n=0}^{\infty} \frac{q^{2 n^{2}}}{\left(q ; q^{2}\right)_{n}}, \\
f_{1}(q)=\sum_{n=0}^{\infty} \frac{q^{n^{2}+n}}{(-q ; q)_{n}}, & \phi_{1}(q)=\sum_{n=0}^{\infty} q^{(n+1)^{2}}\left(-q ; q^{2}\right)_{n}, \\
\psi_{1}(q)=\sum_{n=0}^{\infty} q^{\frac{n(n+1)}{2}}(-q ; q)_{n}, & F_{1}(q)=\sum_{n=0}^{\infty} \frac{q^{2 n(n+1)}}{\left(q ; q^{2}\right)_{n+1}}, \\
\chi_{0}(q)=\sum_{n=0}^{\infty} \frac{q^{n}(q ; q)_{n}}{(q ; q)_{2 n}}
\end{array}
$$

and

$$
\chi_{1}(q)=\sum_{n=0}^{\infty} \frac{q^{n}(q ; q)_{n}}{(q ; q)_{2 n+1}} .
$$

The eighth order mock theta functions of Gordon and McIntosh [3]

and

$$
\begin{aligned}
& S_{0}(q)=\sum_{n=0}^{\infty} \frac{q^{n^{2}}\left(-q ; q^{2}\right)_{n}}{\left(-q^{2} ; q^{2}\right)_{n}}, \\
& T_{0}(q)=\sum_{n=0}^{\infty} \frac{q^{(n+1)(n+2)}\left(-q^{2} ; q^{2}\right)_{n}}{\left(-q ; q^{2}\right)_{n+1}}, \\
& S_{1}(q)=\sum_{n=0}^{\infty} \frac{q^{n^{2}+2 n}\left(-q ; q^{2}\right)_{n}}{\left(-q^{2} ; q^{2}\right)_{n}}, \\
& U_{0}(q)=\sum_{n=0}^{\infty} \frac{q^{n^{2}}\left(-q ; q^{2}\right)_{n}}{\left(-q^{4} ; q^{4}\right)_{n}}, \\
& T_{1}(q)=\sum_{n=0}^{\infty} \frac{q^{n^{2}+n}\left(-q^{2} ; q^{2}\right)_{n}}{\left(-q ; q^{2}\right)_{n+1}} \text {, } \\
& U_{1}(q)=\sum_{n=0}^{\infty} \frac{q^{(n+1)^{2}}\left(-q ; q^{2}\right)_{n}}{\left(-q^{2} ; q^{4}\right)_{n+1}}, \\
& V_{0}(q)=-1+2 \sum_{n=0}^{\infty} \frac{q^{n^{2}}\left(-q ; q^{2}\right)_{n}}{\left(q ; q^{2}\right)_{n}} \\
& =-1+2 \sum_{n=0}^{\infty} \frac{q^{2 n^{2}}\left(-q^{2} ; q^{4}\right)_{n}}{\left(q ; q^{2}\right)_{2 n+1}}
\end{aligned}
$$

$$
\begin{aligned}
V_{1}(q) & =\sum_{n=0}^{\infty} \frac{q^{(n+1)^{2}}\left(-q ; q^{2}\right)_{n}}{\left(q ; q^{2}\right)_{n+1}} \\
& =\sum_{n=0}^{\infty} \frac{q^{2 n^{2}+2 n+1}\left(-q^{4} ; q^{4}\right)_{n}}{\left(q ; q^{2}\right)_{2 n+2}} .
\end{aligned}
$$

\section{Definition of generalized mock theta functions of order five and eight}

B. Srivastava [5] defined generalized functions of fifth order mock theta functions:

$$
f_{0}(t, \alpha, z ; q)=\frac{1}{(t)_{\infty}} \sum_{n=0}^{\infty} \frac{(t)_{n} q^{n^{2}-3 n+n \alpha} z^{2 n}}{(-z ; q)_{n}},
$$




$$
\begin{aligned}
& \phi_{0}(t, \alpha, z ; q)=\frac{1}{(t)_{\infty}} \sum_{n=0}^{\infty} \frac{(t)_{n} q^{n^{2}+n+n \alpha}\left(-\frac{q^{3}}{z^{2}} ; q^{2}\right)_{n}}{z^{2 n}}, \\
& \psi_{0}(t, \alpha, z ; q)=\frac{1}{(t)_{\infty}} \sum_{n=1}^{\infty} \frac{(t)_{n} q^{\frac{n(n+1)}{2}+n \alpha}\left(-\frac{q^{2}}{z} ; q\right)_{n-1}}{z^{n}}, \\
& F_{0}(t, \alpha, z ; q)=\frac{1}{(t)_{\infty}} \sum_{n=0}^{\infty} \frac{(t)_{n} q^{2 n^{2}-5 n+n \alpha} z^{4 n}}{\left(\frac{z^{2}}{q} ; q^{2}\right)_{n}}, \\
& f_{1}(t, \alpha, z ; q)=\frac{1}{(t)_{\infty}} \sum_{n=0}^{\infty} \frac{(t)_{n} q^{n^{2}-2 n+n \alpha} z^{2 n}}{(-z ; q)_{n}}, \\
& \phi_{1}(t, \alpha, z ; q)=\frac{q^{5}}{z^{4}} \frac{1}{(t)_{\infty}} \sum_{n=0}^{\infty} \frac{(t)_{n} q^{n^{2}+3 n+n \alpha}\left(-\frac{q^{3}}{z^{2}} ; q^{2}\right)_{n}}{z^{2 n}}, \\
& \psi_{1}(t, \alpha, z ; q)=\frac{q}{z} \frac{1}{(t)_{\infty}} \sum_{n=0}^{\infty} \frac{(t)_{n} q^{\frac{n(n+1)}{2}+n \alpha}\left(-\frac{q^{2}}{z} ; q\right)_{n}}{z^{n}}, \\
& x_{1}(t, \alpha, z ; q)=\frac{1}{(t)_{\infty}} \sum_{n=0}^{\infty} \frac{(t)_{n} q^{2 n^{2}-3 n+n \alpha} z^{4 n}}{\left(\frac{z^{2}}{q} ; q^{2}\right)_{n+1}}, \\
& (t)_{\infty} \sum_{n=0}^{\infty} \frac{(t)_{n} q^{n \alpha}(z ; q)_{n}}{\left(z^{2} q^{-1} ; q\right)_{2 n}}
\end{aligned}
$$

and

$$
\chi_{1}(t, \alpha, z ; q)=\frac{1}{(t)_{\infty}} \sum_{n=0}^{\infty} \frac{(t)_{n} q^{n \alpha}(z ; q)_{n}}{\left(z^{2} q^{-1} ; q\right)_{2 n+1}} .
$$

For $t=0, \alpha=1$ and $z=q$ these generalized functions reduce to the mock theta functions of order five. Generalization of eighth order mock theta functions:

$$
\begin{aligned}
S_{0}(t, \alpha, z ; q) & =\frac{1}{(t)_{\infty}} \sum_{n=0}^{\infty} \frac{(t)_{n} q^{n^{2}-2 n+n \alpha} z^{n}\left(-z^{2} / q ; q^{2}\right)_{n}}{\left(-z^{2} ; q^{2}\right)_{n}}, \\
S_{1}(t, \alpha, z ; q) & =\frac{1}{(t)_{\infty}} \sum_{n=0}^{\infty} \frac{(t)_{n} q^{n^{2}+n \alpha} z^{n}\left(-z^{2} / q ; q^{2}\right)_{n}}{\left(-z^{2} ; q^{2}\right)_{n}}, \\
T_{0}(t, \alpha, z ; q) & =\frac{1}{(t)_{\infty}} \sum_{n=0}^{\infty} \frac{(t)_{n} q^{n^{2}+n+n \alpha} z^{n+2}\left(-z^{2} ; q^{2}\right)_{n}}{\left(-z^{2} / q ; q^{2}\right)_{n+1}}, \\
T_{1}(t, \alpha, z ; q) & =\frac{1}{(t)_{\infty}} \sum_{n=0}^{\infty} \frac{(t)_{n} q^{n^{2}-n+n \alpha} z^{n}\left(-z^{2} ; q^{2}\right)_{n}}{\left(-z^{2} / q ; q^{2}\right)_{n+1}}, \\
U_{0}(t, \alpha, z ; q) & =\frac{1}{(t)_{\infty}} \sum_{n=0}^{\infty} \frac{(t)_{n} q^{n^{2}-3 n+n \alpha} z^{2 n}\left(-z^{2} / q ; q^{2}\right)_{n}}{\left(-z^{2} q^{2} ; q^{4}\right)_{n}}, \\
U_{1}(t, \alpha, z ; q) & =\frac{1}{(t)_{\infty}} \sum_{n=0}^{\infty} \frac{(t)_{n} q^{n^{2}-n+n \alpha} z^{2 n+1}\left(-z^{2} / q ; q^{2}\right)_{n}}{\left(-z^{2} ; q^{4}\right)_{n+1}}, \\
V_{0}(t, \alpha, z ; q) & =-1+\frac{2}{(t)_{\infty}} \sum_{n=0}^{\infty} \frac{(t)_{n} q^{n^{2}-2 n+n \alpha} z^{n}\left(-z ; q^{2}\right)_{n}}{\left(z^{2} / q ; q^{2}\right)_{n}}
\end{aligned}
$$




$$
=-1+\frac{2}{(t)_{\infty}} \sum_{n=0}^{\infty} \frac{(t)_{n} q^{2 n^{2}-3 n+n \alpha} z^{2 n}\left(-z^{2} ; q^{4}\right)_{n}}{\left(z^{2} / q ; q^{2}\right)_{2 n+1}}
$$

and

$$
\begin{aligned}
V_{1}(t, \alpha, z ; q) & =\frac{1}{(t)_{\infty}} \sum_{n=0}^{\infty} \frac{(t)_{n} q^{n^{2}+n \alpha} z^{n+1}\left(-z ; q^{2}\right)_{n}}{\left(z^{2} / q ; q^{2}\right)_{n+1}}, \\
& =\frac{1}{(t)_{\infty}} \sum_{n=0}^{\infty} \frac{(t)_{n} q^{2 n^{2}-n+n \alpha} z^{2 n+1}\left(-z^{2} q^{2} ; q^{4}\right)_{n}}{\left(z^{2} / q ; q^{2}\right)_{2 n+2}} .
\end{aligned}
$$

For $t=0, \alpha=1$, and $z=q$, these generalized functions reduce to the mock theta functions of order eight.

\section{Definition of bilateral generalized mock theta functions of order third, fifth and eighth}

We shall denote by $f_{c}(t, \alpha, \beta, z ; q)$, the bilateral form of $f(t, \alpha, \beta, z ; q)$ with similar notation for other fuctions. The basic bilateral form of generalized third order mock theta functions [6]:

$$
\begin{aligned}
& f_{c}(t, \alpha, \beta, z ; q)=\frac{1}{(t)_{\infty}} \sum_{n=-\infty}^{\infty} \frac{(t)_{n} q^{n^{2}-4 n+n \beta} \alpha^{n} z^{2 n}}{(-z ; q)_{n}(-\alpha z / q ; q)_{n}}, \\
& \phi_{c}(t, \alpha, \beta, z ; q)=\frac{1}{(t)_{\infty}} \sum_{n=-\infty}^{\infty} \frac{(t)_{n} q^{n^{2}-3 n+n \beta} z^{2 n}}{\left(-\alpha z^{2} / q ; q^{2}\right)_{n}}, \\
& \psi_{c}(t, \alpha, \beta, z ; q)=\frac{1}{(t)_{\infty}} \sum_{n=-\infty}^{\infty} \frac{(t)_{n} q^{n^{2}-n+n \beta} z^{2 n+1}}{\left(\alpha z^{2} / q^{2} ; q^{2}\right)_{n+1}} \\
& \chi_{c}(t, \beta, z ; q)=\frac{1}{(t)_{\infty}} \sum_{n=-\infty}^{\infty} \frac{(t)_{n} q^{n^{2}-3 n+n \beta} z^{2 n}}{(v z ; q)_{n}\left(-v^{2} z ; q\right)_{n}}, \\
& v_{c}(t, \alpha, \beta, z ; q)=\frac{1}{(t)_{\infty}} \sum_{n=-\infty}^{\infty} \frac{(t)_{n} q^{n^{2}-2 n+n \beta} z^{2 n}}{\left(-\alpha^{2} z^{2} / q^{3} ; q^{2}\right)_{n+1}}, \\
& \omega_{c}(t, \alpha, \beta, z ; q)=\frac{1}{(t)_{\infty}} \sum_{n=-\infty}^{\infty} \frac{(t)_{n} q^{2 n^{2}-5 n-4+n \beta} \alpha^{2 n} z^{4(n+1)}}{\left(z^{2} / q ; q^{2}\right)_{n+1}\left(\alpha^{2} z^{2} / q^{3} ; q^{2}\right)_{n+1}}
\end{aligned}
$$

and

$$
\rho_{c}(t, \beta, z ; q)=\frac{z^{4}}{q^{4}(t)_{\infty}} \sum_{n=-\infty}^{\infty} \frac{(t)_{n} q^{2 n^{2}-3 n+n \beta} z^{4 n}}{\left(v^{2} z^{2} / q ; q^{2}\right)_{n+1}\left(v^{-2} z^{2} / q ; q^{2}\right)_{n+1}} .
$$

For $t=0, \beta=1, \alpha=q$ and $z=q$, these bilateral generalized functions reduce to bilateral mock theta functions of order three.

The basic bilateral form of generalized fifth order mock theta functions:

$$
\begin{aligned}
& f_{0 c}(t, \alpha, z ; q)=\frac{1}{(t)_{\infty}} \sum_{n=-\infty}^{\infty} \frac{(t)_{n} q^{n^{2}-3 n+n \alpha} z^{2 n}}{(-z ; q)_{n}}, \\
& \phi_{0 c}(t, \alpha, z ; q)=\frac{1}{(t)_{\infty}} \sum_{n=-\infty}^{\infty} \frac{(t)_{n} q^{n^{2}+n+n \alpha}\left(-\frac{q^{3}}{z^{2}} ; q^{2}\right)_{n}}{z^{2 n}}, \\
& \psi_{0 c}(t, \alpha, z ; q)=\frac{1}{(t)_{\infty}} \sum_{n=-\infty}^{\infty} \frac{(t)_{n} q^{\frac{n(n+1)}{2}+n \alpha}\left(-\frac{q^{2}}{z} ; q\right)_{n-1}}{z^{n}}, \\
& F_{0 c}(t, \alpha, z ; q)=\frac{1}{(t)_{\infty}} \sum_{n=-\infty}^{\infty} \frac{(t)_{n} q^{2 n^{2}-5 n+n \alpha} z^{4 n}}{\left(\frac{z^{2}}{q} ; q^{2}\right)_{n}},
\end{aligned}
$$




$$
\begin{aligned}
& f_{1 c}(t, \alpha, z ; q)=\frac{1}{(t)_{\infty}} \sum_{n=-\infty}^{\infty} \frac{(t)_{n} q^{n^{2}-2 n+n \alpha} z^{2 n}}{(-z ; q)_{n}}, \\
& \phi_{1 c}(t, \alpha, z ; q)=\frac{q^{5}}{z^{4}} \frac{1}{(t)_{\infty}} \sum_{n=-\infty}^{\infty} \frac{(t)_{n} q^{n^{2}+3 n+n \alpha}\left(-\frac{q^{3}}{z^{2}} ; q^{2}\right)_{n}}{z^{2 n}}, \\
& \psi_{1 c}(t, \alpha, z ; q)=\frac{q}{z} \frac{1}{(t)_{\infty}} \sum_{n=-\infty}^{\infty} \frac{(t)_{n} q^{\frac{n(n+1)}{2}+n \alpha}\left(-\frac{q^{2}}{z} ; q\right)_{n}}{z^{n}}, \\
& F_{1 c}(t, \alpha, z ; q)=\frac{1}{(t)_{\infty}} \sum_{n=-\infty}^{\infty} \frac{(t)_{n} q^{2 n^{2}-3 n+n \alpha} z^{4 n}}{\left(\frac{z^{2}}{q} ; q^{2}\right)_{n+1}}, \\
& \chi_{0 c}(t, \alpha, z ; q)=\frac{1}{(t)_{\infty}} \sum_{n=-\infty}^{\infty} \frac{(t)_{n} q^{n \alpha}(z ; q)_{n}}{\left(z^{2} q^{-1} ; q\right)_{2 n}}
\end{aligned}
$$

and

$$
\chi_{1 c}(t, \alpha, z ; q)=\frac{1}{(t)_{\infty}} \sum_{n=-\infty}^{\infty} \frac{(t)_{n} q^{n \alpha}(z ; q)_{n}}{\left(z^{2} q^{-1} ; q\right)_{2 n+1}} .
$$

For $t=0, \alpha=1$, and $z=q$ these bilateral generalized functions reduce to bilateral mock theta functions of order five.

The bilateral form of generalized eighth order mock theta functions:

$$
\begin{aligned}
S_{0 c}(t, \alpha, z ; q) & =\frac{1}{(t)_{\infty}} \sum_{n=-\infty}^{\infty} \frac{(t)_{n} q^{n^{2}-2 n+n \alpha} z^{n}\left(-z^{2} / q ; q^{2}\right)_{n}}{\left(-z^{2} ; q^{2}\right)_{n}}, \\
S_{1 c}(t, \alpha, z ; q) & =\frac{1}{(t)_{\infty}} \sum_{n=-\infty}^{\infty} \frac{(t)_{n} q^{n^{2}+n \alpha} z^{n}\left(-z^{2} / q ; q^{2}\right)_{n}}{\left(-z^{2} ; q^{2}\right)_{n}}, \\
T_{0 c}(t, \alpha, z ; q) & =\frac{1}{(t)_{\infty}} \sum_{n=-\infty}^{\infty} \frac{(t)_{n} q^{n^{2}+n+n \alpha} z^{n+2}\left(-z^{2} ; q^{2}\right)_{n}}{\left(-z^{2} / q ; q^{2}\right)_{n+1}}, \\
T_{1 c}(t, \alpha, z ; q) & =\frac{1}{(t)_{\infty}} \sum_{n=-\infty}^{\infty} \frac{(t)_{n} q^{n^{2}-n+n \alpha} z^{n}\left(-z^{2} ; q^{2}\right)_{n}}{\left(-z^{2} / q ; q^{2}\right)_{n+1}}, \\
U_{0 c}(t, \alpha, z ; q) & =\frac{1}{(t)_{\infty}} \sum_{n=-\infty}^{\infty} \frac{(t)_{n} q^{n^{2}-3 n+n \alpha} z^{2 n}\left(-z^{2} / q ; q^{2}\right)_{n}}{\left(-z^{2} q^{2} ; q^{4}\right)_{n}}, \\
U_{1 c}(t, \alpha, z ; q) & =\frac{1}{(t)_{\infty}} \sum_{n=-\infty}^{\infty} \frac{(t)_{n} q^{n^{2}-n+n \alpha} z^{2 n+1}\left(-z^{2} / q ; q^{2}\right)_{n}}{\left(-z^{2} ; q^{4}\right)_{n+1}}, \\
V_{0 c}(t, \alpha, z ; q) & =-1+\frac{2}{(t)_{\infty}} \sum_{n=-\infty}^{\infty} \frac{(t)_{n} q^{n^{2}-2 n+n \alpha} z^{n}\left(-z ; q^{2}\right)_{n}}{\left(z^{2} / q ; q^{2}\right)_{n}} \\
& =-1+\frac{2}{(t)_{\infty}} \sum_{n=-\infty}^{\infty} \frac{(t)_{n} q^{2 n^{2}-3 n+n \alpha} z^{2 n}\left(-z^{2} ; q^{4}\right)_{n}}{\left(z^{2} / q ; q^{2}\right)_{2 n+1}}
\end{aligned}
$$

and

$$
\begin{aligned}
V_{1 c}(t, \alpha, z ; q) & =\frac{1}{(t)_{\infty}} \sum_{n=-\infty}^{\infty} \frac{(t)_{n} q^{n^{2}+n \alpha} z^{n+1}\left(-z ; q^{2}\right)_{n}}{\left(z^{2} / q ; q^{2}\right)_{n+1}} \\
& =\frac{1}{(t)_{\infty}} \sum_{n=-\infty}^{\infty} \frac{(t)_{n} q^{2 n^{2}-n+n \alpha} z^{2 n+1}\left(-z^{2} q^{2} ; q^{4}\right)_{n}}{\left(z^{2} / q ; q^{2}\right)_{2 n+2}} .
\end{aligned}
$$

For $t=0, \alpha=1$, and $z=q$ these bilateral generalized functions reduce to bilateral mock theta functions of order eight. 


\section{Theorem 1}

\section{Generalized functions are $F_{q}$ - functions}

The bilateral generalized functions of fifth order mock theta functions $f_{0 \mathrm{c}}(t, \alpha, z ; q), \phi_{0 \mathrm{c}}(t, \alpha, z ; q)$, $\psi_{0 \mathrm{c}}(t, \alpha, z ; q), F_{0 \mathrm{c}}(t, \alpha, z ; q), f_{1 \mathrm{c}}(t, \alpha, z ; q), \phi_{1 \mathrm{c}}(t, \alpha, z ; q), \psi_{1 \mathrm{c}}(t, \alpha, z ; q), F_{1 c}(t, \alpha, z ; q), \chi_{0}(t, \alpha, z ; q)$ and $\chi_{1}(t, \alpha, z ; q)$ are $F_{q}$ - functions.

\section{Proofs}

We shall give the proof for $f_{0 c}(t, \alpha, z ; q)$ only. The proof for the other functions are similar, hence omitted. Applying the difference operator $D_{q, t}$ to $f_{0 c}(t, \alpha, z ; q)$, we have

$$
\begin{aligned}
t D_{q, t} f_{0 c}(t, \alpha, z ; q)= & f_{0 c}(t, \alpha, z ; q)-f_{0 c}(t q, \alpha, z ; q) \\
= & \frac{1}{(t)_{\infty}} \sum_{n=-\infty}^{\infty} \frac{(t)_{n} q^{n^{2}-3 n+n \alpha} z^{2 n}}{(-z ; q)_{n}} \\
& -\frac{1}{(t q)_{\infty}} \sum_{n=-\infty}^{\infty} \frac{(t q)_{n} q^{n^{2}-3 n+n \alpha} z^{2 n}}{(-z ; q)_{n}} \\
= & \frac{1}{(t)_{\infty}} \sum_{n=-\infty}^{\infty} \frac{(t)_{n} q^{n^{2}-3 n+n \alpha} z^{2 n}}{(-z ; q)_{n}} \\
& -\frac{1}{(t)_{\infty}} \sum_{n=-\infty}^{\infty} \frac{(t)_{n} q^{n^{2}-3 n+n \alpha} z^{2 n}\left(1-t q^{n}\right)}{(-z ; q)_{n}} \\
= & \frac{t}{(t)_{\infty}} \sum_{n=-\infty}^{\infty} \frac{(t)_{n} q^{n^{2}-3 n+n(\alpha+1)} z^{2 n}}{(-z ; q)_{n}} \\
= & t f_{0 c}(t, \alpha+1, z ; q) .
\end{aligned}
$$

So

$$
D_{q, t} f_{0 c}(t, \alpha, z ; q)=f_{0 c}(t, \alpha+1, z ; q) .
$$

Hence $f_{0 c}(t, \alpha, z ; q)$ is a $F_{q}$ - function.

As stated earlier the proofs for other functions are similar, so omitted.

\section{Theorem 2}

The generalized bilateral mock theta functions of order eight $S_{0 \mathrm{c}}(t, \alpha, z ; q), S_{1 \mathrm{c}}(t, \alpha, z ; q), T_{0 \mathrm{c}}(t, \alpha, z ; q), T_{1 \mathrm{c}}(t, \alpha, z ; q), U_{0 \mathrm{c}}(t, \alpha, z ; q), U_{1 \mathrm{c}}(t, \alpha, z ; q), V_{0 \mathrm{c}}(t, \alpha, z ; q)$ and $V_{1 \mathrm{c}}(t, \alpha, z ; q)$ are $F_{q}$ - functions.

\section{Proofs}

We shall give the proof for $S_{0 \mathrm{c}}(t, \alpha, z ; q)$ only. The proof for the other functions are similar, hence omitted. Applying the difference operator $D_{q, t}$ to $S_{0 \mathrm{c}}(t, \alpha, z ; q)$, we have

$$
\begin{aligned}
t D_{q, t} S_{0 \mathrm{c}}(t, \alpha, z ; q)= & S_{0 \mathrm{c}}(t, \alpha, z ; q)-S_{0 \mathrm{c}}(t q, \alpha, z ; q) \\
& =\frac{1}{(t)_{\infty}} \sum_{n=-\infty}^{\infty} \frac{(t)_{n} q^{n^{2}-2 n+n \alpha} z^{n}\left(-z^{2} / q ; q^{2}\right)_{n}}{\left(-z^{2} ; q^{2}\right)_{n}}
\end{aligned}
$$




$$
\begin{aligned}
& \quad-\frac{1}{(t q)_{\infty}} \sum_{n=-\infty}^{\infty} \frac{(t q)_{n} q^{n^{2}-2 n+n \alpha} z^{n}\left(-z^{2} / q ; q^{2}\right)_{n}}{\left(-z^{2} ; q^{2}\right)_{n}} \\
& =\frac{1}{(t)_{\infty}} \sum_{n=-\infty}^{\infty} \frac{(t)_{n} q^{n^{2}-2 n+n \alpha} z^{n}\left(-z^{2} / q ; q^{2}\right)_{n}}{\left(-z^{2} ; q^{2}\right)_{n}} \\
& \quad-\frac{1}{(t)_{\infty}} \sum_{n=-\infty}^{\infty} \frac{(t)_{n} q^{n^{2}-2 n+n \alpha} z^{n}\left(-z^{2} / q ; q^{2}\right)_{n}\left(1-t q^{n}\right)}{\left(-z^{2} ; q^{2}\right)_{n}} \\
& =\frac{t}{(t)_{\infty}} \sum_{n=-\infty}^{\infty} \frac{(t)_{n} q^{n^{2}-2 n+n(\alpha+1)} z^{n}\left(-z^{2} / q ; q^{2}\right)_{n}}{\left(-z^{2} ; q^{2}\right)_{n}} \\
& =t S_{0 \mathrm{c}}(t, \alpha+1, z ; q) .
\end{aligned}
$$

So

$$
D_{q, t} S_{0 \mathrm{c}}(t, \alpha, z ; q)=S_{0 \mathrm{c}}(t, \alpha+1, z ; q) .
$$

Hence $S_{0 c}(t, \alpha, z ; q)$ is a $F_{q}$ - function.

As stated earlier the proofs for other functions are similar, so omitted.

\section{Alternative definition of generalized bilateral mock theta}

\section{Functions of order five and eight}

We shall use the following bilateral transformations of Bailey [7] to give alternative definitions.

(i) ${ }_{2} \psi_{2}\left[\begin{array}{l}a, b \\ c, d\end{array} ; q ; Z\right]=\frac{(a Z, d / a, c / b, d q / a b Z ; q)_{\infty}}{(Z, d, q / b, c d / a b Z ; q)_{\infty}}{ }_{2} \psi_{2}\left[\begin{array}{c}a, a b Z / d \\ a Z, c\end{array} ; q ; d / a\right]$

(ii) ${ }_{2} \psi_{2}\left[\begin{array}{l}a, b \\ c, d\end{array} ; q ; Z\right]=\frac{(a Z, b Z, c q / a b Z, d q / a b z ; q)_{\infty}}{(q / a, q / b, c, d ; q)_{\infty}} \psi_{2}\left[\begin{array}{c}a b Z / c, a b Z / d ; q ; c d / a b Z \\ a z, b Z\end{array}\right]$

7.1 Alternative definition of bilateral generalized functions associated with fifth order mock theta functions

(i)Making $a, b \rightarrow \infty, c=-z, d=0, z=\frac{z^{2} q^{\alpha}}{a b q^{2}}$ in (49), we get after a little simplification

$$
f_{0 c}(\alpha, z ; q)=\frac{\left(-q^{3} / z q^{\alpha} ; q\right)_{\infty}}{(-z ; q)_{\infty}} \sum_{n=-\infty}^{\infty} q^{\frac{n^{2}-n}{2}} z^{n}\left(-z q^{\alpha} / q^{2} ; q\right)_{n} .
$$

Similarly we have the following definitions. We have given in brackets the value of parameters taken in each case.

(ii) $\quad \phi_{1 c}(\alpha, z ; q)=\frac{q^{5}\left(-q^{\alpha+4} / z^{2} ; q^{2}\right)_{\infty}}{z^{4}\left(-z^{2} / q ; q^{2}\right)_{\infty}} \sum_{n=-\infty}^{\infty} \frac{q^{2 n^{2}+5 n+n \alpha}}{z^{4 n}\left(-q^{\alpha+4} / z^{2} ; q^{2}\right)_{n}}$.

$$
\left[q \rightarrow q^{2}, a \rightarrow \infty, b=-\frac{q^{3}}{z^{2}}, c, d=0, z=-\frac{q^{\alpha+4}}{a z^{2}}\right]
$$

(iii) $\quad \psi_{0 c}(\alpha, z ; q)=\frac{q^{\alpha+1}\left(-q^{\alpha+2} / z ; q\right)_{\infty}}{z(-z / q ; q)_{\infty}} \sum_{n=-\infty}^{\infty} \frac{q^{n^{2}+3 n+n \alpha}}{z^{2 n}\left(-q^{\alpha+2} / z ; q\right)_{n}}$.

$$
\left[a \rightarrow \infty, b=-\frac{q^{2}}{z}, c, d=0, z=-\frac{q^{\alpha+2}}{a z}\right]
$$

(iv) $\quad F_{0 c}(\alpha, z ; q)=\frac{\left(q^{4} / z^{2} q^{\alpha} ; q^{2}\right)_{\infty}}{\left(z^{2} / q ; q^{2}\right)_{\infty}} \sum_{n=-\infty}^{\infty}(-1)^{n} q^{n^{2}-2 n} z^{2 n}\left(z^{2} q^{\alpha} / q^{2} ; q^{2}\right)_{n}$.

$$
\left[q \rightarrow q^{2}, a, b \rightarrow \infty, c=\frac{z^{2}}{q}, d=0, z=\frac{z^{4} q^{\alpha}}{a b q^{3}}\right]
$$


(v)

$$
\begin{aligned}
f_{1 c}(\alpha, z ; q)=\frac{\left(-q^{2} / z q^{\alpha} ; q\right)_{\infty}}{(-z ; q)_{\infty}} \sum_{n=-\infty}^{\infty} q^{\frac{n^{2}-n}{2}} z^{n}\left(-z q^{\alpha} / q ; q\right)_{n} \\
{\left[a, b \rightarrow \infty, c=-z, d=0, z=\frac{z^{2} q^{\alpha}}{a b q}\right] }
\end{aligned}
$$

(vi) $\quad \phi_{0 c}(\alpha, z ; q)=\frac{\left(-q^{\alpha+2} / z^{2} ; q^{2}\right)_{\infty}}{\left(-z^{2} / q ; q^{2}\right)_{\infty}} \sum_{n=-\infty}^{\infty} \frac{q^{2 n^{2}+3 n+n \alpha}}{z^{4 n}\left(-q^{\alpha+2} / z^{2} ; q^{2}\right)_{n}}$.

$$
\left[q \rightarrow q^{2}, a \rightarrow \infty, b=-\frac{q^{3}}{z^{2}}, c, d=0, z=-\frac{q^{\alpha+2}}{a z^{2}}\right]
$$

(vii) $\quad \psi_{1 c}(\alpha, z ; q)=\frac{q\left(-q^{\alpha+1} / z ; q\right)_{\infty}}{z(-z / q ; q)_{\infty}} \sum_{n=-\infty}^{\infty} \frac{q^{n^{2}+2 n+n \alpha}}{z^{2 n}\left(-q^{\alpha+1} / z ; q\right)_{n}}$.

$$
\left[a \rightarrow \infty, b=-\frac{q^{2}}{z}, c, d=0, z=-\frac{q^{\alpha+1}}{a z}\right]
$$

(viii) $\quad F_{1 c}(\alpha, z ; q)=\frac{\left(q^{4} / z^{2} q^{\alpha} ; q^{2}\right)_{\infty}}{\left(1-z^{2} / q\right)\left(z^{2} q ; q^{2}\right)_{\infty}} \sum_{n=-\infty}^{\infty}(-1)^{n} q^{n^{2}} z^{2 n}\left(z^{2} q^{\alpha} / q^{2} ; q^{2}\right)_{n}$.

$$
\left[q \rightarrow q^{2}, a, b \rightarrow \infty, c=z^{2} q, d=0, z=\frac{z^{4} q^{\alpha}}{a b q}\right]
$$

7.2 Alternative definition of bilateral generalized functions associated with eighth order mock theta functions

By using (48), we have the following alternative definitions:

(i) $S_{0 c}(\alpha, z ; q)=\frac{\left(-z q^{\alpha} / q, q ; q^{2}\right)_{\infty}}{\left(-q^{3} / z^{2} ; q^{2}\right)_{\infty}} \sum_{n=-\infty}^{\infty} \frac{q^{2 n^{2}-4 n+n \alpha} z^{3 n}}{\left(-z^{2},-z q^{\alpha} / q ; q^{2}\right)_{n}}$.

$$
\left[q \rightarrow q^{2}, a \rightarrow \infty, b=\frac{-z^{2}}{q}, c=-z^{2}, d=0, z=\frac{-z q^{\alpha}}{a q}\right]
$$

(ii) $\quad S_{1 c}(\alpha, z ; q)=\frac{\left(-z q^{\alpha+1}, q ; q^{2}\right)_{\infty}}{\left(-q^{3} / z^{2} ; q^{2}\right)_{\infty}} \sum_{n=-\infty}^{\infty} \frac{q^{2 n^{2}-2 n+n \alpha} z^{3 n}}{\left(-z^{2},-z q^{\alpha+1} ; q^{2}\right)_{n}}$.

$$
\left[q \rightarrow q^{2}, a \rightarrow \infty, b=\frac{-z^{2}}{q}, c=-z^{2}, d=0, z=\frac{-z q^{\alpha+1}}{a}\right]
$$

(iii) $\quad T_{0 c}(\alpha, z ; q)=\frac{z^{2}\left(-z q^{\alpha+2}, q ; q^{2}\right)_{\infty}}{\left(1+z^{2} / q\right)\left(-q^{2} / z^{2} ; q^{2}\right)_{\infty}} \sum_{n=-\infty}^{\infty} \frac{q^{2 n^{2}+n \alpha} z^{3 n}}{\left(-z^{2} q,-z q^{\alpha+2} ; q^{2}\right)_{n}}$.

$$
\left[q \rightarrow q^{2}, a \rightarrow \infty, b=-z^{2}, c=-z^{2} q, d=0, z=\frac{-z q^{\alpha+2}}{a}\right]
$$

(iv) $\quad T_{1 c}(\alpha, z ; q)=\frac{\left(-z q^{\alpha}, q ; q^{2}\right)_{\infty}}{\left(1+z^{2} / q\right)\left(-q^{2} / z^{2} ; q^{2}\right)_{\infty}} \sum_{n=-\infty}^{\infty} \frac{q^{2 n^{2}-2 n+n \alpha} z^{3 n}}{\left(-z^{2} q,-z q^{\alpha} ; q^{2}\right)_{n}}$.

$$
\left[q \rightarrow q^{2}, a \rightarrow \infty, b=-z^{2}, c=-z^{2} q, d=0, z=\frac{-z q^{\alpha}}{a}\right]
$$

(v) $V_{0 c}(\alpha, z ; q)=-1+2 \frac{\left(-z q^{\alpha} / q,-z / q ; q^{2}\right)_{\infty}}{\left(-q^{2} / z ; q^{2}\right)_{\infty}} \sum_{n=-\infty}^{\infty} \frac{q^{2 n^{2}-3 n+n \alpha} z^{2 n}}{\left(z^{2} / q,-z q^{\alpha} / q ; q^{2}\right)_{n}}$.

$$
\left[q \rightarrow q^{2}, a \rightarrow \infty, b=-z, c=\frac{z^{2}}{q}, d=0, z=\frac{-z q^{\alpha}}{a q}\right]
$$


(vi)

$$
\begin{aligned}
V_{1 c}(\alpha, z ; q)= & \frac{z\left(-z q^{\alpha+1},-z q ; q^{2}\right)_{\infty}}{\left(1-z^{2} / q\right)\left(-q^{2} / z ; q^{2}\right)_{\infty}} \sum_{n=-\infty}^{\infty} \frac{q^{2 n^{2}-n+n \alpha} z^{2 n}}{\left(z^{2} q,-z q^{\alpha+1} ; q^{2}\right)_{n}} . \\
& {\left[q \rightarrow q^{2}, a \rightarrow \infty, b=-z, c=z^{2} q, d=0, z=\frac{-z q^{\alpha+1}}{a}\right] }
\end{aligned}
$$

VIII. Relations between generalized bilateral mock theta functions

8.1 Relations among generalized basic bilateral fifth order mock theta functions

$$
\begin{aligned}
& f_{0 c}\left(2, q^{3} / z^{2} ; q^{2}\right)=\frac{\left(-z^{2} / q ; q^{2}\right)_{\infty}}{\left(-q^{3} / z^{2} ; q^{2}\right)_{\infty}} \phi_{0 c}(1, z ; q) . \\
& {\left[q \rightarrow q^{2}, z=\frac{q^{3}}{z^{2}}, \alpha=2 \text { in (50) and using (29) }\right] }
\end{aligned}
$$

(ii)

$$
\begin{aligned}
\psi_{0 c}\left(1, q^{3} / z ; q\right)= & \frac{z(-z ; q)_{\infty}}{q\left(-q^{2} / z ; q\right)_{\infty}} f_{0 c}(1, z ; q) . \\
& {\left[z=\frac{q^{3}}{z}, \alpha=1\right. \text { in (52) and using (28)] }}
\end{aligned}
$$

$$
\begin{aligned}
& F_{0 c}\left(1, i q^{2} / z ; q\right)=\frac{\left(-z^{2} / q ; q^{2}\right)_{\infty}}{\left(-q^{3} / z^{2} ; q^{2}\right)_{\infty}} \phi_{0 c}(1, z ; q) \text {. } \\
& {\left[z=\frac{i q^{2}}{z}, \alpha=1\right. \text { in (53) and using (29)] }}
\end{aligned}
$$

$$
\begin{aligned}
f_{1 c}\left(1, q^{2} / z ; q\right)= & \frac{z(-z / q ; q)_{\infty}}{q\left(-q^{2} / z ; q\right)_{\infty}} \psi_{0 c}(0, z ; q) . \\
& {\left[z=\frac{q^{2}}{z}, \alpha=1 \text { in (54) and using (30) }\right] }
\end{aligned}
$$

$$
\begin{aligned}
\phi_{0 c}\left(1, i q^{2} / z ; q\right) & =\frac{\left(z^{2} / q ; q^{2}\right)_{\infty}}{\left(q^{3} / z^{2} ; q^{2}\right)_{\infty}} F_{0 c}(1, z ; q) . \\
& {\left[z=\frac{i q^{2}}{z}, \alpha=1 \text { in (55) and using (31) }\right] }
\end{aligned}
$$

$$
\begin{aligned}
\psi_{1 c}\left(1, q^{2} / z ; q\right) & =\frac{z(-z ; q)_{\infty}}{q(-q / z ; q)_{\infty}} f_{1 c}(1, z ; q) . \\
& {\left[\begin{array}{l}
z=\frac{q^{2}}{z}, \alpha=1 \text { in (56) and using (32) }
\end{array}\right] }
\end{aligned}
$$

$$
\begin{array}{r}
F_{1 c}\left(1, i q^{2} / z ; q\right)=\frac{z^{4}\left(-z^{2} / q ; q^{2}\right)_{\infty}}{q^{5}\left(1+q^{3} / z^{2}\right)\left(-q^{5} / z^{2} ; q^{2}\right)_{\infty}} \phi_{1 c}(1, z ; q) . \\
{\left[\begin{array}{l}
\left.z=\frac{i q^{2}}{z}, \alpha=1 \text { in (57) and using (33) }\right]
\end{array}\right.}
\end{array}
$$

8.2 Relations between generalized basic bilateral eighth and third order mock theta functions

$$
\begin{aligned}
S_{0 c}(1, z ; q) & =\frac{\left(-z, q ; q^{2}\right)_{\infty}}{\left(-q^{3} / z^{2} ; q^{2}\right)_{\infty}} f_{c}\left(q^{2} / z, 3 / 2, z^{2} ; q^{2}\right) . \\
& {\left[\alpha=1 \text { in }(58) \text { and } q \rightarrow q^{2}, z \rightarrow z^{2}, \alpha=q^{2} / z, \beta=3 / 2 \text { in (21) }\right] }
\end{aligned}
$$

(ii) $S_{0 c}(1, z ; q)=\frac{q^{6}\left(1+z / q^{2}\right)\left(1+z^{2} / q^{2}\right)\left(-z, q ; q^{2}\right)_{\infty}}{z^{4}\left(-q^{3} / z^{2} ; q^{2}\right)_{\infty}} \omega_{c}\left(q / z^{1 / 2}, 2, i z / q^{1 / 2} ; q\right)$.

$$
\left[\alpha=1 \text { in (58) and } z=i z / q^{1 / 2}, \alpha=q / z^{1 / 2}, \beta=2\right. \text { in (26) ] }
$$

(iii)

$$
\begin{aligned}
& S_{1 c}(1, z ; q)=\frac{\left(-z q^{2}, q ; q^{2}\right)_{\infty}}{\left(-q^{3} / z^{2} ; q^{2}\right)_{\infty}} f_{c}\left(q^{4} / z, 3 / 2, z^{2} ; q^{2}\right) . \\
& {\left[\alpha=1 \text { in }(59) \text { and } q \rightarrow q^{2}, z \rightarrow z^{2}, \alpha=q^{4} / z, \beta=3 / 2 \text { in (21) }\right]}
\end{aligned}
$$


(iv) $S_{1 c}(1, z ; q)=\frac{q^{6}(1+z)\left(1+z^{2} / q^{2}\right)\left(-z q^{2}, q ; q^{2}\right)_{\infty}}{z^{4}\left(-q^{3} / z^{2} ; q^{2}\right)_{\infty}} \omega_{c}\left(q^{2} / z^{1 / 2}, 2, i z / q^{1 / 2} ; q\right)$.

$$
\left[\alpha=1 \text { in (59) and } z=i z / q^{1 / 2}, \alpha=q^{2} / z^{1 / 2}, \beta=2 \text { in (26) }\right]
$$

(v) $\quad T_{0 c}(1, z ; q)=\frac{z^{2}\left(-z q^{3}, q ; q^{2}\right)_{\infty}}{\left(1+z^{2} / q\right)\left(-q^{2} / z^{2} ; q^{2}\right)_{\infty}} f_{c}\left(q^{4} / z, 3 / 2, z^{2} q ; q^{2}\right)$.

$$
\left[\alpha=1 \text { in (60) and } q \rightarrow q^{2}, z \rightarrow z^{2} q, \alpha=q^{4} / z, \beta=3 / 2\right. \text { in (21) ] }
$$

$$
\begin{aligned}
& T_{0 c}(1, z ; q)=\frac{q^{4}(1+z q)\left(-z q^{3}, q ; q^{2}\right)_{\infty}}{z^{2}\left(-q^{2} / z^{2} ; q^{2}\right)_{\infty}} \omega_{c}\left(q^{2} / z^{\frac{1}{2}}, 2, i z ; q\right) . \\
& {\left[\alpha=1 \text { in }(60) \text { and } z=i z, \alpha=q^{2} / z^{\frac{1}{2}}, \beta=2 \text { in }(26)\right]}
\end{aligned}
$$

(vii) $\quad T_{1 c}(1, z ; q)=\frac{\left(-z q, q ; q^{2}\right)_{\infty}}{\left(1+z^{2} / q\right)\left(-q^{2} / z^{2} ; q^{2}\right)_{\infty}} f_{c}\left(q^{2} / z, 3 / 2, z^{2} q ; q^{2}\right)$.

$$
\left[\alpha=1 \text { in (61) and } q \rightarrow q^{2}, z \rightarrow z^{2} q, \alpha=q^{2} / z, \beta=3 / 2\right. \text { in (21) ] }
$$

$$
\begin{aligned}
T_{1 c}(1, z ; q) & =\frac{q^{4}(1+z / q)\left(-z q, q ; q^{2}\right)_{\infty}}{z^{4}\left(-q^{2} / z^{2} ; q^{2}\right)_{\infty}} \omega_{c}\left(q / z^{1 / 2}, 2, i z ; q\right) . \\
{[\alpha} & \left.=1 \text { in }(61) \text { and } z=i z, \alpha=q / z^{1 / 2}, \beta=2 \text { in (26) }\right] .
\end{aligned}
$$

\section{Expansions of generalized bilateral mock theta functions}

Using the general transformation of Slater [7, p. 129 (5.4.3)], we express the generalized functions in a bilateral series. The advantage of using this transformation is that the $c^{\prime} s$ are absent on the left hand side and we can choose them conveniently. For $r=2$, we have the transformation

$$
\begin{aligned}
\frac{\left(b_{1}, b_{2}, q / a_{1}, q / a_{2}, d Z, q / d Z ; q\right)_{\infty}}{\left(c_{1}, c_{2}, q / c_{1}, q / c_{2} ; q\right)_{\infty}}{ }_{2} \psi_{2}\left[\begin{array}{l}
a_{1}, a_{2} \\
b_{1}, b_{2} ; q ; z
\end{array}\right] \\
=\frac{q}{c_{1}} \frac{\left(c_{1} / a_{1}, c_{1} / a_{2}, q b_{1} / c_{1}, q b_{2} / c_{1}, d c_{1} z / q, q^{2} / d c_{1} z ; q\right)_{\infty}}{\left(c_{1}, q / c_{1}, c_{1} / c_{2}, q c_{2} / c_{1} ; q\right)_{\infty}} \\
\times{ }_{2} \psi_{2}\left[\begin{array}{l}
q a_{1} / c_{1}, q a_{2} / c_{1} \\
q b_{1} / c_{1}, q b_{2} / c_{1} ; q ; z
\end{array}\right]+\operatorname{idem}\left(c_{1} ; c_{2}\right)
\end{aligned}
$$

where $d=a_{1} a_{2} / c_{1} c_{2},\left|b_{1} b_{2} / a_{1} a_{2}\right|<|z|<1$, and idem $\left(c_{1} ; c_{2}\right)$ after the expression means that the proceeding expression is repeated with $c_{1}$ and $c_{2}$ interchanged.

\subsection{Expansions for generalized bilateral fifth order mock theta functions}

(i)Making $a_{1}, a_{2} \rightarrow \infty$ and taking $b_{1}=-z, b_{2}=0, z=\frac{z^{2} q^{\alpha}}{q^{2} a_{1} a_{2}}$ in (64), we have

$$
\begin{aligned}
\frac{\left(-z, z^{2} q^{\alpha} / c_{1} c_{2} q^{2}, c_{1} c_{2} q^{3} / z^{2} q^{\alpha} ; q\right)_{\infty}}{\left(c_{1}, c_{2}, q / c_{1}, q / c_{2} ; q\right)_{\infty}} f_{0 c}(\alpha, z ; q) & =\frac{q}{c_{1}} \frac{\left(-q z / c_{1}, z^{2} q^{\alpha} / c_{2} q^{3}, c_{2} q^{4} / z^{2} q^{\alpha} ; q\right)_{\infty}}{\left(c_{1}, q / c_{1}, c_{1} / c_{2}, q c_{2} / c_{1} ; q\right)_{\infty}} \\
& \times \sum_{n=-\infty}^{\infty} \frac{q^{n^{2}-n+n \alpha} z^{2 n}}{c_{1}^{2 n}\left(-q z / c_{1} ; q\right)_{n}}+\operatorname{idem}\left(c_{1} ; c_{2}\right) .
\end{aligned}
$$

(ii) Similarly we obtain the expansions for the other functions, the value of the parameters is given in parentheses.

$$
\begin{array}{r}
\frac{\left(-z^{2} / q, q^{\alpha+5} / c_{1} c_{2} z^{4}, c_{1} c_{2} z^{4} / q^{\alpha+3} ; q^{2}\right)_{\infty}}{\left(c_{1}, c_{2}, q^{2} / c_{1}, q^{2} / c_{2} ; q^{2}\right)_{\infty}} \phi_{0 c}(\alpha, z ; q) \\
=\frac{q^{2}}{c_{1}} \frac{\left(-c_{1} z^{2} / q^{3}, q^{\alpha+3} / c_{2} z^{4}, c_{2} z^{4} / q^{\alpha+1} ; q^{2}\right)_{\infty}}{\left(c_{1}, q^{2} / c_{1}, c_{1} / c_{2}, q^{2} c_{2} / c_{1} ; q^{2}\right)_{\infty}} \\
\quad \times \sum_{n=-\infty}^{\infty} \frac{q^{n^{2}+3 n+n \alpha}\left(-q^{5} / z^{2} c_{1} ; q^{2}\right)_{n}}{c_{1}^{n} z^{2 n}}+\operatorname{idem}\left(c_{1} ; c_{2}\right) . \\
\left(q \rightarrow q^{2}, a_{1} \rightarrow \infty, a_{2}=\frac{-q^{3}}{z^{2}}, b_{1}=0, b_{2}=0, z=\frac{-q^{\alpha+2}}{z^{2} a_{1}}\right)
\end{array}
$$


(iii) $\frac{\left(-z / q, q^{\alpha+4} / c_{1} c_{2} z^{2}, c_{1} c_{2} z^{2} / q^{\alpha+3} ; q\right)_{\infty}}{\left(c_{1}, c_{2}, q / c_{1}, q / c_{2} ; q\right)_{\infty}} \psi_{0 c}(\alpha, z ; q)$

$$
\begin{array}{r}
=\frac{q^{\alpha+2}}{z c_{1}} \frac{\left(-c_{1} z / q^{2}, q^{\alpha+3} / c_{2} z^{2}, c_{2} z^{2} / q^{\alpha+2} ; q\right)_{\infty}}{\left(c_{1}, q / c_{1}, c_{1} / c_{2}, q c_{2} / c_{1} ; q\right)_{\infty}} \\
\times \sum_{n=-\infty}^{\infty} \frac{q^{\frac{n^{2}+5 n}{2}+n \alpha}\left(-q^{3} / z c_{1} ; q\right)_{n}}{c_{1}^{n} z^{n}}+\operatorname{idem}\left(c_{1} ; c_{2}\right) . \\
\left(a_{1} \rightarrow \infty, a_{2}=\frac{-q^{2}}{z} b_{1}=0, b_{2}=0, z=\frac{-q^{\alpha+2}}{z a_{1}}\right)
\end{array}
$$

(iv) $\frac{\left(z^{2} / q, z^{4} q^{\alpha} / c_{1} c_{2} q^{3}, c_{1} c_{2} q^{5} / z^{4} q^{\alpha} ; q^{2}\right)_{\infty}}{\left(c_{1}, c_{2}, q^{2} / c_{1}, q^{2} / c_{2} ; q^{2}\right)_{\infty}} F_{0 c}(\alpha, z ; q)$

$$
\begin{gathered}
=\frac{q^{2}}{c_{1}} \frac{\left(z^{2} q / c_{1}, z^{4} q^{\alpha} / c_{2} q^{5}, c_{2} q^{7} / q^{\alpha} z^{4} ; q^{2}\right)_{\infty}}{\left(c_{1}, q^{2} / c_{1}, c_{1} / c_{2}, q^{2} c_{2} / c_{1} ; q^{2}\right)_{\infty}} \\
\times \sum_{n=-\infty}^{\infty} \frac{q^{2 n^{2}-n+n \alpha} z^{4 n}}{c_{1}^{2 n}\left(z^{2} q / c_{1} ; q^{2}\right)_{n}}+\operatorname{idem}\left(c_{1} ; c_{2}\right) . \\
\left(q \rightarrow q^{2}, a_{1}, a_{2} \rightarrow \infty, b_{1}=\frac{z^{2}}{q}, b_{2}=0, z=\frac{z^{4} q^{\alpha}}{q^{3} a_{1} a_{2}}\right)
\end{gathered}
$$

(v) $\frac{\left(-z, z^{2} q^{\alpha} / c_{1} c_{2} q, c_{1} c_{2} q^{2} / z^{2} q^{\alpha} ; q\right)_{\infty}}{\left(c_{1}, c_{2}, q / c_{1}, q / c_{2} ; q\right)_{\infty}} f_{1 c}(\alpha, z ; q)$

$$
\begin{gathered}
=\frac{q}{c_{1}} \frac{\left(-q z / c_{1}, z^{2} q^{\alpha} / c_{2} q^{2}, c_{2} q^{3} / z^{2} q^{\alpha} ; q\right)_{\infty}}{\left(c_{1}, q / c_{1}, c_{1} / c_{2}, q c_{2} / c_{1} ; q\right)_{\infty}} \\
\quad \times \sum_{n=-\infty}^{\infty} \frac{q^{n^{2}+n \alpha} z^{2 n}}{c_{1}^{2 n}\left(-q z / c_{1} ; q\right)_{n}}+\operatorname{idem}\left(c_{1} ; c_{2}\right) . \\
\left(a_{1}, a_{2} \rightarrow \infty, b_{1}=-z, b_{2}=0, z=\frac{z^{2} q^{\alpha}}{q a_{1} a_{2}}\right)
\end{gathered}
$$

(vi) $\frac{\left(-z^{2} / q, q^{\alpha+7} / c_{1} c_{2} z^{4}, c_{1} c_{2} z^{4} / q^{\alpha+5} ; q^{2}\right)_{\infty}}{\left(c_{1}, c_{2}, q^{2} / c_{1}, q^{2} / c_{2} ; q^{2}\right)_{\infty}} \phi_{1 c}(\alpha, z ; q)$

$$
\begin{aligned}
&= \frac{q^{7}}{z^{4} c_{1}} \frac{\left(-c_{1} z^{2} / q^{3}, q^{\alpha+5} / c_{2} z^{4}, c_{2} z^{4} / q^{\alpha+3} ; q^{2}\right)_{\infty}}{\left(c_{1}, q^{2} / c_{1}, c_{1} / c_{2}, q^{2} c_{2} / c_{1} ; q^{2}\right)_{\infty}} \\
& \times \sum_{n=-\infty}^{\infty} \frac{q^{n^{2}+5 n+n \alpha}\left(-q^{5} / z^{2} c_{1} ; q^{2}\right)_{n}}{c_{1}^{n} z^{2 n}}+\operatorname{idem}\left(c_{1} ; c_{2}\right) . \\
&\left(q \rightarrow q^{2}, a_{1} \rightarrow \infty, a_{2}=\frac{-q^{3}}{z^{2}}, b_{1}=0, b_{2}=0, z=\frac{-q^{\alpha+4}}{z^{2} a_{1}}\right)
\end{aligned}
$$

(vii) $\frac{\left(-z / q, q^{\alpha+3} / c_{1} c_{2} z^{2}, c_{1} c_{2} z^{2} / q^{\alpha+2} ; q\right)_{\infty}}{\left(c_{1}, c_{2}, q / c_{1}, q / c_{2} ; q\right)_{\infty}} \psi_{1 c}(\alpha, z ; q)$

$$
\begin{aligned}
= & \frac{q^{2}}{z c_{1}} \frac{\left(-c_{1} z / q^{2}, q^{\alpha+2} / c_{2} z^{2}, c_{2} z^{2} / q^{\alpha+1} ; q\right)_{\infty}}{\left(c_{1}, q / c_{1}, c_{1} / c_{2}, q c_{2} / c_{1} ; q\right)_{\infty}} \\
& \times \sum_{n=-\infty}^{\infty} \frac{q^{\frac{n^{2}+3 n}{2}+n \alpha}\left(-q^{3} / z c_{1} ; q\right)_{n}}{c_{1}^{n} z^{n}}+\operatorname{idem}\left(c_{1} ; c_{2}\right) . \\
\left(a_{1} \rightarrow \infty, a_{2}=\right. & \left.\frac{-q^{2}}{z}, b_{1}=0, b_{2}=0, z=\frac{-q^{\alpha+1}}{z a_{1}}\right)
\end{aligned}
$$


(viii) $\frac{\left(z^{2} q, z^{4} q^{\alpha} / c_{1} c_{2} q, c_{1} c_{2} q^{3} / z^{4} q^{\alpha} ; q^{2}\right)_{\infty}}{\left(c_{1}, c_{2}, q^{2} / c_{1}, q^{2} / c_{2} ; q^{2}\right)_{\infty}} F_{1 c}(\alpha, z ; q)$

$$
\begin{gathered}
=\frac{q^{2}}{c_{1}} \frac{\left(z^{2} q^{3} / c_{1}, z^{4} q^{\alpha} / c_{2} q^{3}, c_{2} q^{5} / q^{\alpha} z^{4} ; q^{2}\right)_{\infty}}{\left(c_{1}, q^{2} / c_{1}, c_{1} / c_{2}, q^{2} c_{2} / c_{1} ; q^{2}\right)_{\infty}} \\
\times \frac{1}{\left(1-z^{2} / q\right)} \sum_{n=-\infty}^{\infty} \frac{q^{2 n^{2}+n+n \alpha} z^{4 n}}{c_{1}^{2 n}\left(z^{2} q^{3} / c_{1} ; q^{2}\right)_{n}}+\operatorname{idem}\left(c_{1} ; c_{2}\right) . \\
\left(q \rightarrow q^{2}, a_{1}, a_{2} \rightarrow \infty, b_{1}=z^{2} q, b_{2}=0, z=\frac{z^{4} q^{\alpha}}{q a_{1} a_{2}}\right)
\end{gathered}
$$

\subsection{Expansions for generalized bilateral eighth order mock theta functions}

(i) $\frac{\left(-z^{2},-q^{3} / z^{2}, z^{3} q^{\alpha} / c_{1} c_{2} q^{2}, c_{1} c_{2} q^{4} / z^{3} q^{\alpha} ; q^{2}\right)_{\infty}}{\left(c_{1}, c_{2}, q^{2} / c_{1}, q^{2} / c_{2} ; q^{2}\right)_{\infty}} \mathrm{S}_{0 c}(\alpha, z ; q)$

$$
\begin{gathered}
=\frac{q^{2}}{c_{1}} \frac{\left(-c_{1} q / z^{2},-z^{2} q^{2} / c_{1}, z^{3} q^{\alpha} / c_{2} q^{4}, c_{2} q^{6} / z^{3} q^{\alpha} ; q^{2}\right)_{\infty}}{\left(c_{1}, q^{2} / c_{1}, c_{1} / c_{2}, q^{2} c_{2} / c_{1} ; q^{2}\right)_{\infty}} \\
\times \sum_{n=-\infty}^{\infty} \frac{q^{n^{2}+n \alpha} z^{n}\left(-z^{2} q / c_{1}, q^{2}\right)_{n}}{c_{1}^{n}\left(-z^{2} q^{2} / c_{1} ; q^{2}\right)_{n}}+\operatorname{idem}\left(c_{1} ; c_{2}\right) . \\
\left(q \rightarrow q^{2}, a_{1} \rightarrow \infty, a_{2}=\frac{-z^{2}}{q}, b_{1}=-z^{2}, b_{2}=0, z=\frac{-z q^{\alpha}}{a_{1} q}\right)
\end{gathered}
$$

(ii) $\frac{\left(-z^{2},-q^{3} / z^{2}, z^{3} q^{\alpha} / c_{1} c_{2}, c_{1} c_{2} q^{2} / z^{3} q^{\alpha} ; q^{2}\right)_{\infty}}{\left(c_{1}, c_{2}, q^{2} / c_{1}, q^{2} / c_{2} ; q^{2}\right)_{\infty}} \mathrm{S}_{1 c}(\alpha, z ; q)$

$$
\begin{gathered}
=\frac{q^{2}}{c_{1}} \frac{\left(-c_{1} q / z^{2},-z^{2} q^{2} / c_{1}, z^{3} q^{\alpha} / c_{2} q^{2}, c_{2} q^{4} / z^{3} q^{\alpha} ; q^{2}\right)_{\infty}}{\left(c_{1}, q^{2} / c_{1}, c_{1} / c_{2}, q^{2} c_{2} / c_{1} ; q^{2}\right)_{\infty}} \\
\times \sum_{n=-\infty}^{\infty} \frac{q^{n^{2}+2 n+n \alpha} z^{n}\left(-z^{2} q / c_{1}, q^{2}\right)_{n}}{c_{1}^{n}\left(-z^{2} q^{2} / c_{1} ; q^{2}\right)_{n}}+\operatorname{idem}\left(c_{1} ; c_{2}\right) . \\
\left(q \rightarrow q^{2}, a_{1} \rightarrow \infty, a_{2}=\frac{-z^{2}}{q}, b_{1}=-z^{2}, b_{2}=0, z=\frac{-z q^{\alpha+1}}{a_{1}}\right)
\end{gathered}
$$

(iii) $\frac{\left(-z^{2} q,-q^{2} / z^{2}, z^{3} q^{\alpha+2} / c_{1} c_{2}, c_{1} c_{2} / z^{3} q^{\alpha} ; q^{2}\right)_{\infty}}{\left(c_{1}, c_{2}, q^{2} / c_{1}, q^{2} / c_{2} ; q^{2}\right)_{\infty}} T_{0 c}(\alpha, z ; q)$

$$
\begin{aligned}
&= \frac{q^{2}}{c_{1}} \frac{\left(-c_{1} / z^{2},-z^{2} q^{3} / c_{1}, z^{3} q^{\alpha} / c_{2}, c_{2} q^{2} / z^{3} q^{\alpha} ; q^{2}\right)_{\infty}}{\left(c_{1}, q^{2} / c_{1}, c_{1} / c_{2}, q^{2} c_{2} / c_{1} ; q^{2}\right)_{\infty}} \\
& \times \frac{z^{2}}{\left(1+z^{2} / q\right)} \sum_{n=-\infty}^{\infty} \frac{q^{n^{2}+3 n+n \alpha} z^{n}\left(-z^{2} q^{2} / c_{1}, q^{2}\right)_{n}}{c_{1}^{n}\left(-z^{2} q^{3} / c_{1} ; q^{2}\right)_{n}}+\operatorname{idem}\left(c_{1} ; c_{2}\right) . \\
&\left(q \rightarrow q^{2}, a_{1} \rightarrow \infty, a_{2}=-z^{2}, b_{1}=-z^{2} q, b_{2}=0, z=\frac{-z q^{\alpha+2}}{a_{1}}\right)
\end{aligned}
$$

(iv) $\frac{\left(-z^{2} q,-q^{2} / z^{2}, z^{3} q^{\alpha} / c_{1} c_{2}, c_{1} c_{2} q^{2} / z^{3} q^{\alpha} ; q^{2}\right)_{\infty}}{\left(c_{1}, c_{2}, q^{2} / c_{1}, q^{2} / c_{2} ; q^{2}\right)_{\infty}} T_{1 c}(\alpha, z ; q)$

$$
\begin{gathered}
=\frac{q^{2}}{c_{1}} \frac{\left(-c_{1} / z^{2},-z^{2} q^{3} / c_{1}, z^{3} q^{\alpha} / c_{2} q^{2}, c_{2} q^{4} / z^{3} q^{\alpha} ; q^{2}\right)_{\infty}}{\left(c_{1}, q^{2} / c_{1}, c_{1} / c_{2}, q^{2} c_{2} / c_{1} ; q^{2}\right)_{\infty}} \\
\quad \times \frac{1}{\left(1+z^{2} / q\right)} \sum_{n=-\infty}^{\infty} \frac{q^{n^{2}+n+n \alpha} z^{n}\left(-z^{2} q^{2} / c_{1}, q^{2}\right)_{n}}{c_{1}^{n}\left(-z^{2} q^{3} / c_{1} ; q^{2}\right)_{n}}+\operatorname{idem}\left(c_{1} ; c_{2}\right) . \\
\left(q \rightarrow q^{2}, a_{1} \rightarrow \infty, a_{2}=-z^{2}, b_{1}=-z^{2} q, b_{2}=0, z=\frac{-z q^{\alpha}}{a_{1}}\right)
\end{gathered}
$$


(v) $\frac{\left(z^{2} / q,-q^{2} / z, z^{2} q^{\alpha} / c_{1} c_{2} q, c_{1} c_{2} q^{3} / z^{2} q^{\alpha} ; q^{2}\right)_{\infty}}{\left(c_{1}, c_{2}, q^{2} / c_{1}, q^{2} / c_{2} ; q^{2}\right)_{\infty}}\left[\frac{V_{0 c}(\alpha, z ; q)+1}{2}\right]$

$$
\begin{gathered}
=\frac{q^{2}}{c_{1}} \frac{\left(-c_{1} / z, z^{2} q / c_{1}, z^{2} q^{\alpha} / c_{2} q^{3}, c_{2} q^{5} / z^{2} q^{\alpha} ; q^{2}\right)_{\infty}}{\left(c_{1}, q^{2} / c_{1}, c_{1} / c_{2}, q^{2} c_{2} / c_{1} ; q^{2}\right)_{\infty}} \\
\times \sum_{n=-\infty}^{\infty} \frac{q^{n^{2}+n \alpha} z^{n}\left(-z q^{2} / c_{1}, q^{2}\right)_{n}}{c_{1}^{n}\left(z^{2} q / c_{1} ; q^{2}\right)_{n}}+\operatorname{idem}\left(c_{1} ; c_{2}\right) . \\
\left(q \rightarrow q^{2}, a_{1} \rightarrow \infty, a_{2}=-z, b_{1}=z^{2} / q, b_{2}=0, z=\frac{-z q^{\alpha}}{q a_{1}}\right)
\end{gathered}
$$

(vi) $\frac{\left(z^{2} q,-q^{2} / z, z^{2} q^{\alpha+1} / c_{1} c_{2}, c_{1} c_{2} q / z^{2} q^{\alpha} ; q^{2}\right)_{\infty}}{\left(c_{1}, c_{2}, q^{2} / c_{1}, q^{2} / c_{2} ; q^{2}\right)_{\infty}} V_{1 c}(\alpha, z ; q)$

$$
\begin{aligned}
= & \frac{q^{2}}{c_{1}} \frac{\left(-c_{1} / z, z^{2} q^{3} / c_{1}, z^{2} q^{\alpha} / c_{2} q, c_{2} q^{3} / z^{2} q^{\alpha} ; q^{2}\right)_{\infty}}{\left(c_{1}, q^{2} / c_{1}, c_{1} / c_{2}, q^{2} c_{2} / c_{1} ; q^{2}\right)_{\infty}} \\
& \times \frac{z}{\left(1-z^{2} / q\right)} \sum_{n=-\infty}^{\infty} \frac{q^{n^{2}+2 n+n \alpha} z^{n}\left(-z q^{2} / c_{1}, q^{2}\right)_{n}}{c_{1}^{n}\left(z^{2} q^{3} / c_{1} ; q^{2}\right)_{n}}+\operatorname{idem}\left(c_{1} ; c_{2}\right) . \\
(q \rightarrow & \left.q^{2}, a_{1} \rightarrow \infty, a_{2}=-z, b_{1}=z^{2} q, b_{2}=0, z=\frac{-z q^{\alpha+1}}{a_{1}}\right)
\end{aligned}
$$

\section{Relations between bilateral generalized mock theta functions}

10.1 Relations among bilateral generalized functions associated with fifth order mock theta functions

$$
\begin{aligned}
& \text { (i) } \frac{\left(-z, z^{2} q^{\alpha} / c_{2} q^{4}, c_{2} q^{5} / z^{2} q^{\alpha} ; q\right)_{\infty}}{\left(q^{2}, 1 / q, c_{2}, q / c_{2} ; q\right)_{\infty}} f_{0 c}(\alpha, z ; q) \\
& =\frac{1}{q} \frac{\left(-z / q, z^{2} q^{\alpha} / c_{2} q^{3}, c_{2} q^{4} / z^{2} q^{\alpha} ; q\right)_{\infty}}{\left(q^{2}, 1 / q, q^{2} / c_{2}, c_{2} / q ; q\right)_{\infty}} f_{1 c}\left(\alpha-1, \frac{z}{q} ; q\right)+\operatorname{idem}\left(q^{2} ; c_{2}\right) . \\
& \left(z=\frac{z}{q}, \alpha=\alpha-1 \text { in (32) and taking } c_{1}=q^{2}\right. \text { in (65)) } \\
& \text { (ii) } \frac{\left(-z^{2} / q, q^{\alpha+4} / c_{2} z^{4}, c_{2} z^{4} / q^{\alpha+2} ; q^{2}\right)_{\infty}}{\left(q, q, c_{2}, q^{2} / c_{2} ; q^{2}\right)_{\infty}} \phi_{0 c}(\alpha, z ; q)=\frac{z^{2}\left(-z^{2} / q^{2}, q^{\alpha+3} / c_{2} z^{4}, c_{2} z^{4} / q^{\alpha+1} ; q^{2}\right)_{\infty}}{q^{\alpha}\left(q, q, q / c_{2}, q c_{2} ; q^{2}\right)_{\infty}} \\
& \times \psi_{0 c}\left(\frac{\alpha-1}{2}, z^{2} ; q^{2}\right)+\operatorname{idem}\left(q ; c_{2}\right) . \\
& \left(q \rightarrow q^{2}, z=z^{2}, \alpha=\frac{\alpha-1}{2} \text { in (30) and taking } c_{1}=q\right. \text { in (66)) } \\
& \text { (iii) } \frac{\left(-z^{2} / q, q^{\alpha+4} / c_{2} z^{4}, c_{2} z^{4} / q^{\alpha+2} ; q^{2}\right)_{\infty}}{\left(q, q, c_{2}, q^{2} / c_{2} ; q^{2}\right)_{\infty}} \phi_{0 c}(\alpha, z ; q)=\frac{z^{4}\left(-z^{2} / q^{2}, q^{\alpha+3} / c_{2} z^{4}, c_{2} z^{4} / q^{\alpha+1} ; q^{2}\right)_{\infty}}{q^{5}\left(q, q, q / c_{2}, q c_{2} ; q^{2}\right)_{\infty}} \\
& \times \phi_{1 c}\left(\alpha-2, \frac{z}{q^{1 / 2}} ; q\right)+\operatorname{idem}\left(q ; c_{2}\right) . \\
& \left(z=\frac{z}{q^{1 / 2}}, \alpha=\alpha-2 \text { in (33) and taking } c_{1}=q \text { in (66) }\right) \\
& \text { (iv) } \frac{\left(-z / q, q^{\alpha+2} / c_{2} z^{2}, c_{2} z^{2} / q^{\alpha+1} ; q\right)_{\infty}}{\left(q^{2}, 1 / q, c_{2}, q / c_{2} ; q\right)_{\infty}} \psi_{0 c}(\alpha, z ; q)=\frac{q^{\alpha}\left(-z, q^{\alpha+3} / c_{2} z^{2}, c_{2} z^{2} / q^{\alpha+2} ; q\right)_{\infty}}{\left(q^{2}, 1 / q, q^{2} / c_{2}, c_{2} / q ; q\right)_{\infty}} \\
& \times \psi_{1 c}(\alpha+1, z q ; q)+\operatorname{idem}\left(q^{2} ; c_{2}\right) . \\
& \left(z=z q, \alpha=\alpha+1 \text { in (34) and taking } c_{1}=q^{2}\right. \text { in (67)) } \\
& \text { (v) } \frac{\left(z^{2} / q, z^{4} q^{\alpha} / c_{2} q^{4}, c_{2} q^{6} / z^{4} q^{\alpha} ; q^{2}\right)_{\infty}}{\left(q, q, c_{2}, q^{2} / c_{2} ; q^{2}\right)_{\infty}} F_{0 c}(\alpha, z ; q)=\frac{q\left(z^{2}, z^{4} q^{\alpha} / c_{2} q^{5}, c_{2} q^{7} / q^{\alpha} z^{4} ; q^{2}\right)_{\infty}}{\left(q, q, q / c_{2}, q c_{2} ; q^{2}\right)_{\infty}} \\
& \times f_{0 c}\left(\frac{\alpha+3}{2},-z^{2} ; q^{2}\right)+\operatorname{idem}\left(q ; c_{2}\right) .
\end{aligned}
$$




$$
\left(q \rightarrow q^{2}, z=-z^{2}, \alpha=\frac{\alpha+3}{2} \text { in (28) and taking } c_{1}=q \text { in (68) }\right)
$$

(vi) $\frac{\left(z^{2} / q, z^{4} q^{\alpha} / c_{2} q^{4}, c_{2} q^{6} / z^{4} q^{\alpha} ; q^{2}\right)_{\infty}}{\left(q, q, c_{2}, q^{2} / c_{2} ; q^{2}\right)_{\infty}} F_{0 c}(\alpha, z ; q)=\frac{q\left(z^{2}, z^{4} q^{\alpha} / c_{2} q^{5}, c_{2} q^{7} / q^{\alpha} z^{4} ; q^{2}\right)_{\infty}}{\left(1-z^{2} / q^{2}\right)\left(q, q, q / c_{2}, q c_{2} ; q^{2}\right)_{\infty}}$

$$
\times F_{1 c}\left(\alpha+2, \frac{z}{q^{1 / 2}} ; q\right)+\operatorname{idem}\left(q ; c_{2}\right) \text {. }
$$

$$
\left(z=\frac{z}{q^{1 / 2}}, \alpha=\alpha+2 \text { in (35) and taking } c_{1}=q \text { in (68) }\right)
$$

(vii) $\frac{\left(-z^{2} / q, q^{\alpha+6} / c_{2} z^{4}, c_{2} z^{4} / q^{\alpha+4} ; q^{2}\right)_{\infty}}{\left(q, q, c_{2}, q^{2} / c_{2} ; q^{2}\right)_{\infty}} \phi_{1 c}(\alpha, z ; q)=\frac{q^{5}}{z^{3}} \frac{\left(-z^{2} / q^{2}, q^{\alpha+5} / c_{2} z^{4}, c_{2} z^{4} / q^{\alpha+3} ; q^{2}\right)_{\infty}}{\left(q, q, q / c_{2}, q c_{2} ; q^{2}\right)_{\infty}}$

$$
\times \psi_{1 c}\left(\frac{\alpha+3}{2}, z^{2} ; q^{2}\right)+\operatorname{idem}\left(q ; c_{2}\right) .
$$

$$
\left(q \rightarrow q^{2}, z=z^{2}, \alpha=\frac{\alpha+3}{2} \text { in (34) and taking } c_{1}=q\right. \text { in (70)) }
$$

(viii) $\frac{\left(z^{2} q, z^{4} q^{\alpha} / c_{2} q^{4}, c_{2} q^{6} / z^{4} q^{\alpha} ; q^{2}\right)_{\infty}}{\left(q^{3}, 1 / q, c_{2}, q^{2} / c_{2} ; q^{2}\right)_{\infty}} F_{1 c}(\alpha, z ; q)=\frac{1}{q} \frac{\left(z^{2}, z^{4} q^{\alpha} / c_{2} q^{3}, c_{2} q^{5} / q^{\alpha} z^{4} ; q^{2}\right)_{\infty}}{\left(q^{3}, 1 / q, q^{3} / c_{2}, c_{2} / q ; q^{2}\right)_{\infty}}$

$$
\times \frac{1}{\left(1-z^{2} / q\right)} f_{1 c}\left(\frac{\alpha-1}{2},-z^{2} ; q^{2}\right)+\operatorname{idem}\left(q^{3} ; c_{2}\right) .
$$

$$
\left(q \rightarrow q^{2}, z=-z^{2}, \alpha=\frac{\alpha-1}{2} \text { in (32) and taking } c_{1}=q^{3}\right. \text { in (72)) }
$$

10.2 Relations among generalized bilateral functions associated with eighth order mock theta functions

(i) $\frac{\left(-z^{2},-q^{3} / z^{2}, z^{3} q^{\alpha} / c_{2} q^{3}, c_{2} q^{5} / z^{3} q^{\alpha} ; q^{2}\right)_{\infty}}{\left(1+z^{2} / q\right)\left(q, q, c_{2}, q^{2} / c_{2} ; q^{2}\right)_{\infty}} \mathrm{S}_{0 c}(\alpha, z ; q)$

$$
\begin{aligned}
& =\frac{q\left(-q^{2} / z^{2},-z^{2} q, z^{3} q^{\alpha} / c_{2} q^{4}, c_{2} q^{6} / z^{3} q^{\alpha} ; q^{2}\right)_{\infty}}{\left(q, q, q / c_{2}, q c_{2} ; q^{2}\right)_{\infty}} \mathrm{T}_{1 c}(\alpha, z ; q) \\
& + \text { idem }\left(q ; c_{2}\right) .
\end{aligned}
$$

(ii) $\frac{\left(-z^{2},-q^{3} / z^{2}, z^{3} q^{\alpha} / c_{2} q, c_{2} q^{3} / z^{3} q^{\alpha} ; q^{2}\right)_{\infty}}{\left(1+z^{2} / q\right)\left(q, q, c_{2}, q^{2} / c_{2} ; q^{2}\right)_{\infty}} \mathrm{S}_{1 c}(\alpha, z ; q)$

$$
\begin{gathered}
=\frac{q\left(-q^{2} / z^{2},-z^{2} q, z^{3} q^{\alpha} / c_{2} q^{2}, c_{2} q^{4} / z^{3} q^{\alpha} ; q^{2}\right)_{\infty}}{z^{2}\left(q, q, q / c_{2}, q c_{2} ; q^{2}\right)_{\infty}} \mathrm{T}_{0 c}(\alpha, z ; q) \\
+\operatorname{idem}\left(q ; c_{2}\right) .
\end{gathered}
$$

(Taking $c_{1}=q$ in (74) and using (40))

(iii) $\frac{\left(1+z^{2} / q\right)\left(-z^{2} q,-q^{2} / z^{2}, z^{3} q^{\alpha} / c_{2} q, q^{3} c_{2} / z^{3} q^{\alpha} ; q^{2}\right)_{\infty}}{z^{2}\left(q^{3}, 1 / q, c_{2}, q^{2} / c_{2} ; q^{2}\right)_{\infty}} T_{0 c}(\alpha, z ; q)$

$$
\begin{gathered}
=\frac{\left(-q^{3} / z^{2},-z^{2}, z^{3} q^{\alpha} / c_{2}, c_{2} q^{2} / z^{3} q^{\alpha} ; q^{2}\right)_{\infty}}{q\left(q^{3}, 1 / q, q^{3} / c_{2}, c_{2} / q ; q^{2}\right)_{\infty}} \mathrm{S}_{1 c}(\alpha, z ; q) \\
+\operatorname{idem}\left(q^{3} ; c_{2}\right) .
\end{gathered}
$$

(Taking $c_{1}=q^{3}$ in (75) and using (39))

(iv) $\frac{\left(1+z^{2} / q\right)\left(-z^{2} q,-q^{2} / z^{2}, z^{3} q^{\alpha} / c_{2} q^{3}, q^{5} c_{2} / z^{3} q^{\alpha} ; q^{2}\right)_{\infty}}{\left(q^{3}, 1 / q, c_{2}, q^{2} / c_{2} ; q^{2}\right)_{\infty}} T_{1 c}(\alpha, z ; q)$

$$
\begin{gathered}
=\frac{\left(-q^{3} / z^{2},-z^{2}, z^{3} q^{\alpha} / c_{2} q^{2}, c_{2} q^{4} / z^{3} q^{\alpha} ; q^{2}\right)_{\infty}}{q\left(q^{3}, 1 / q, q^{3} / c_{2}, c_{2} / q ; q^{2}\right)_{\infty}} \mathrm{S}_{0 c}(\alpha, z ; q) \\
\quad+\operatorname{idem}\left(q^{3} ; c_{2}\right) .
\end{gathered}
$$

(Taking $c_{1}=q^{3}$ in (76) and using (38)) 


\section{Conclusion}

We have defined and considered the bilateral form of the generalized fifth and eighth order mock theta functions. I feel it will be helpful in understanding the mock theta functions. Moreover we can have other functions for other values of the parameters.

\section{Acknowledgement}

I am thankful to Dr. Bhaskar Srivastava for his help and guidance.

\section{References}

[1] G.N. Watson, The final problem: An account of the mock theta functions, J. London Math. Soc. 11, 1936, 55-80.

[2] G.E. Andrews, The fifth and the seventh order mock theta functions, Trans. Amer. Math. Soc. 293, 1986, $113-134$

[3] B. Gordon and R. J. McIntosh, Some eight order mock theta functions, J. London Math. Soc. 62, 2000, $321-335$.

[4] G.E. Andrews and B.C. Berndt, Ramanujan's 'Lost' Notebook Part I, Springer New York (2005).

[5] B. Srivastava, Certain bilateral basic hypergeometric transformations and mock theta functions, Hiroshima Math. J. 29, 1999, 19-26.

[6] S. Saba, A study of a generalization of Ramanujan's sixth order and third order mock theta functions, App. Math. 2(5), 2012, 157165.

[7] G. Gasper and M. Rahman, Basic hypergeometric series, Cambridge University Press, Cambridge, (1990). 